\title{
НАРУШЕНИЯ УГЛЕВОДНОГО ОБМЕНА, АССОЦИИРОВАННЫЕ C COVID-19
}

\author{
Кононенко И.В., Шестакова М.В., Калмыкова З.А., Мокрышева Н.Г., Дедов И.И. \\ ФГБУ «НМИЦ эндокринологии» Минздрава России, Москва
}

Вирусная инфекция может является причиной развития сахарного диабета (СД), в том числе активно изучается ее вклад в развитие аутоиммунного СД. Результаты опубликованных с момента начала пандемии COVID-19 исследований демонстрируют нередко впервые зафиксированную гипергликемию у пациентов с данной инфекцией. По-прежнему остается открытым вопрос - наблюдаемые изменения гликемии носят транзиторный или перманентный характер и, если COVID-19 может приводить к развитию СД, то к какому типу будет относиться диабет в этом случае? В связи с этим актуальным является изучение состояния углеводного обмена у пациентов на фоне новой коронавирусной инфекции и оценка наблюдаемых изменений гликемии в динамике. Результаты многочисленных исследований позволяют выделить следующие возможные причины развития гипергликемии при COVID-19: стресс-индуцированную (транзиторную) гипергликемию, исходно не диагностированный сахарный диабет или предиабет, лечение глюкокортикостероидными препаратами, развитие панкреатита вследствие COVID-19. Учитывая особенности репродукции SARS-CoV-2, можно предположить, что развитие СД вследствие новой коронавирусной инфекцией может быть связано с повреждающим действием вируса SARS-Cov-2 в отношении бета-клеток поджелудочной железы (прямое повреждение вирусом, а также вследствие снижения протективного действия ангиотензина 1-7 при связывании вируса с рецептором АПФ2), с повреждением бета-клеток вследствие цитокинового шторма, а также с усилением инсулинорезистентности на фоне активного воспалительного процесса. Нельзя исключить возможность развитие аутоиммунного СД.

Результаты собственных наблюдений 155 пациентов, госпитализированных в ФГБУ «НМИЦ эндокринологии» МЗ РФ с клинико-лабораторной картиной COVID-19 и двусторонней полисегментарной вирусной пневмонией свидетельствуют о том, что только у 42,6\% пациентов все показатели углеводного обмена (гликированный гемоглобин $\left(\mathrm{HbA}_{1}\right)$, глюкоза плазмы натощак (ГПН), глюкоза крови при поступлении (ГКПП) были в норме. СД встречался у 21,9\% пациентов, из них у 16,7\% имелся СД в анамнезе, у 5,2\% диагноз СД2 был поставлен впервые. У оставшихся 35,5\% госпитализированных больных, не имевших СД в анамнезе, отмечалось повышение уровня $\mathrm{HbA}_{1 c}>6,0 \%$ при нормальных показателях ГПН и ГКПП. Стресс-индуцированной гипергликемии зарегистрировано не было. Отмечалась значимая положительная корреляция уровня $\mathrm{HbA}_{1 c}$ с маркерами воспаления (СОЭ, СРБ, ИЛ-6) и отрицательная - с SpO2. Наиболее тяжелое течение заболевания наблюдалось у пациентов $\mathrm{c} \mathrm{HbA}$ 1c $\geq 6,5 \%$ без предшествующего СД в анамнезе.

Через год после выписки из стационара у пациентов без нарушений углеводного обмена на фоне COVID-19 (n=32) показатели ГПН и НbA ${ }_{1 c}$ оставались в пределах нормы. Развитие СД (повышение $\mathrm{HbA}_{1 c}$ $\geq 6,5 \%)$ отмечалось у 4 из 20 пациентов с исходным значением $\mathrm{HbA}_{1 c}>6,0$ и $<6,5 \%$. Таким образом, можно предположить, что SARS-CoV2 обладает диабетогенными свойствами и ускоряет развитие СД у лиц с высоким риском. Повышение НbA1с у пациентов с новой коронавирусной инфекцией может рассматриваться как прогностический фактор в отношении тяжести заболевания, его длительности и исходов, а также указывать на необходимость дальнейшего наблюдения в связи с высоким риском развития СД у данных пациентов. (Исследование выполнено при поддержке компании ООО «Новартис Фарма»).

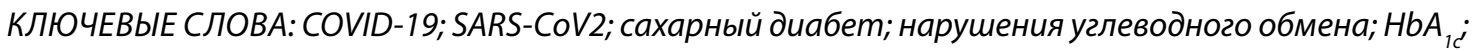

